\title{
An unusual complication following a shoulder relocation attempt in a case of Larsen syndrome: Deep soft tissue laceration of the arm
}

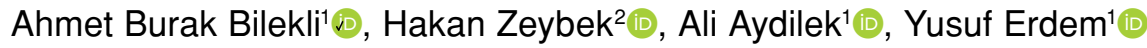 \\ ${ }^{1}$ Department of Orthopedics and Traumatology, Gülhane Training and Research Hospital, Ankara, Turkey \\ ${ }^{2}$ Department of Orthopedics and Traumatology, Atatürk Training and Research Hospital, Izmir, Turkey
}

A traumatic shoulder dislocation (TSD) is one of the most frequent joint dislocations encountered in the orthopedic emergency setting. Its incidence is about 8.2 to $24 / 100,000$ persons per year and complication rates increase up to $26 \%$. [1] Complications include bone and soft tissue injuries such as the labral lesions, glenoid rim fractures, and the Hill Sachs lesions, greater tuberosity fractures, rotator cuff tears; vascular injuries such as rupture, thrombosis or pseudoaneurysm of the axillary or subclavian artery and vein; neurological injuries such as the axillary, suprascapular, musculocutaneous, radial and ulnar nerves; and other rare injuries. ${ }^{[2]}$

Larsen syndrome (LS), with an estimated incidence of $1 / 100,000$ to 250,000 live births, is a rare genetic

Received: October 17, 2021

Accepted: December 20, 2021

Published online: December 22, 2021

Correspondence: Ahmet Burak Bilekli, MD. Gülhane Eğitim ve Araştırma Hastanesi Ortopedi ve Travmatoloji Kliniği,

06010 Keçiören, Ankara, Türkiye.

E-mail: draburakbilekli@yahoo.com

Doi: $10.52312 /$ jdrscr. 2022.3

Citation: Bilekli AB, Zeybek $H$, Aydilek A, Erdem Y. An unusual complication following a shoulder relocation attempt in a case of Larsen syndrome: Deep soft tissue laceration of the arm. Jt Dis Relat Surg Case Rep 2022;1(1):7-10.

(C2022 All right reserved by the Turkish Joint Diseases Foundation

This is an open access article under the terms of the Creative Commons Attribution-NonCommercial License, which permits use, distribution and reproduction in any medium, provided the original work is properly cited and is not used for commercial purposes (http://creativecommons.org/licenses/by-nc/4.0/).

https://www.casereportsjointdrs.org

\section{ABSTRACT}

Traumatic shoulder dislocation is one of the most frequent joint dislocations encountered in the orthopedic emergency setting. A 32-year-old female patient with Larsen syndrome was admitted to our emergency department with an acute left traumatic shoulder dislocation and deep soft tissue laceration of the arm. Her medical history revealed two unsuccessful reduction attempts without sedation in an external center, the second resulting in a laceration. Her shoulder was relocated easily with the Milch maneuver following sedative anesthesia. Intraoperative fluoroscopy control revealed no osseous complications after reduction. Postoperatively, the left shoulder was supported with an arm sling, and the patient was instructed to use it for three weeks while being allowed to do passive range of motion exercises. In conclusion, although rare, closed reduction methods in Larsen syndrome require attention and awareness to abstain from severe complications.

Keywords: Complication, filamin B mutation, Larsen syndrome, reduction, shoulder dislocation, soft tissue laceration.

disorder characterized by typical facial abnormalities and multiple congenital large joint dislocations. ${ }^{[3-5]}$ The current literature about the joint dislocations in LS mainly focuses on the treatment modalities for the congenital dislocations, particularly on the hip and knee, which may be the most common orthopedic manifestation in LS. ${ }^{[5]}$ The literature reveals little information about the shoulder pathologies seen in the patients with LS, and "subluxable" shoulders are the main common presentation. To the best of our knowledge, none of the previous reports presented TSD in an LS patient.

Herein, we report clinical and radiological features of an anterior TSD in a 32-year-old female patient with LS and an unusual complication of the shoulder 
relocation attempts; i.e. deep soft tissue laceration of the arm.

\section{CASE REPORT}

A 32-year-old female patient was admitted to our emergency department not only with acute left TSD, but also a large and deep soft tissue laceration of the arm (Figure 1). Her shoulder was dislocated after a fall from her own height on her extended upper extremity at home. She was initially admitted to an external center where the diagnosis of anterior TSD was made. Two unsuccessful reduction attempts were performed in the emergency room (ER) and the second reduction attempt resulted in a laceration. The patient informed us that she was offered an orthopedics and traumatology consultation; however, she refused treatment and follow-up in that center; therefore, she was admitted to our ER $4 \mathrm{~h}$ after dislocation. Upon inspection, her left arm was abducted and externally rotated and she had a dressing on the posterior aspect of the arm. She resisted range of motion (ROM) examination due to pain. There was squaring of the shoulder. After removing the dressing, an approximately 20 -cm skin and subcutaneous tissue laceration, exposing the fat and muscle tissues at the medial, posterior, and lateral aspects of the mid-arm was observed with no active bleeding. Her left shoulder was first dislocated after a fall at home approximately one year ago and was relocated successfully in another ER, without sedation. She was diagnosed with LS in another institution during her early childhood. The patient had characteristic features suggestive of LS such as hypertelorism, proptotic eyes, low nose bridge, macrocephalus, short neck length, short stature, and bilateral pes equinovarus deformity (Figure 2).

Cephazolin, tetanus prophylaxis, and $50 \mathrm{mg}$ of intramuscular tramadol were administered in the ER after the examination. A shoulder anteroposterior $\mathrm{X}$-ray and computed tomography were obtained which revealed an anterior TSD locked at the anterior glenoid rim due to Hill-Sachs lesion. The preoperative routine complete blood count, blood chemistry, liver function, and coagulation studies were normal. A written informed consent was obtained from the patient and she was admitted to the operating room. Her shoulder was relocated easily with the Milch maneuver following sedative anesthesia. Intraoperative fluoroscopy control revealed no osseous complications after reduction. The wound was irrigated with saline and wound edges were infiltrated with prilocaine. The subcutaneous and skin tissue layers were closed in a routine fashion (Figure 3). Postoperatively, the left shoulder was supported with an arm sling, and the patient was instructed to use it for three weeks while being allowed to do passive ROM exercises. There were no neurological complications during early follow-up.

Given the rare nature of this syndrome, the patient's past medical file was requested from her sister the day after surgery. Her medical file revealed that she had a positive genetic test result for filamin B (FLNB) gene mutation and, therefore, no further genetic or laboratory tests were performed to confirm
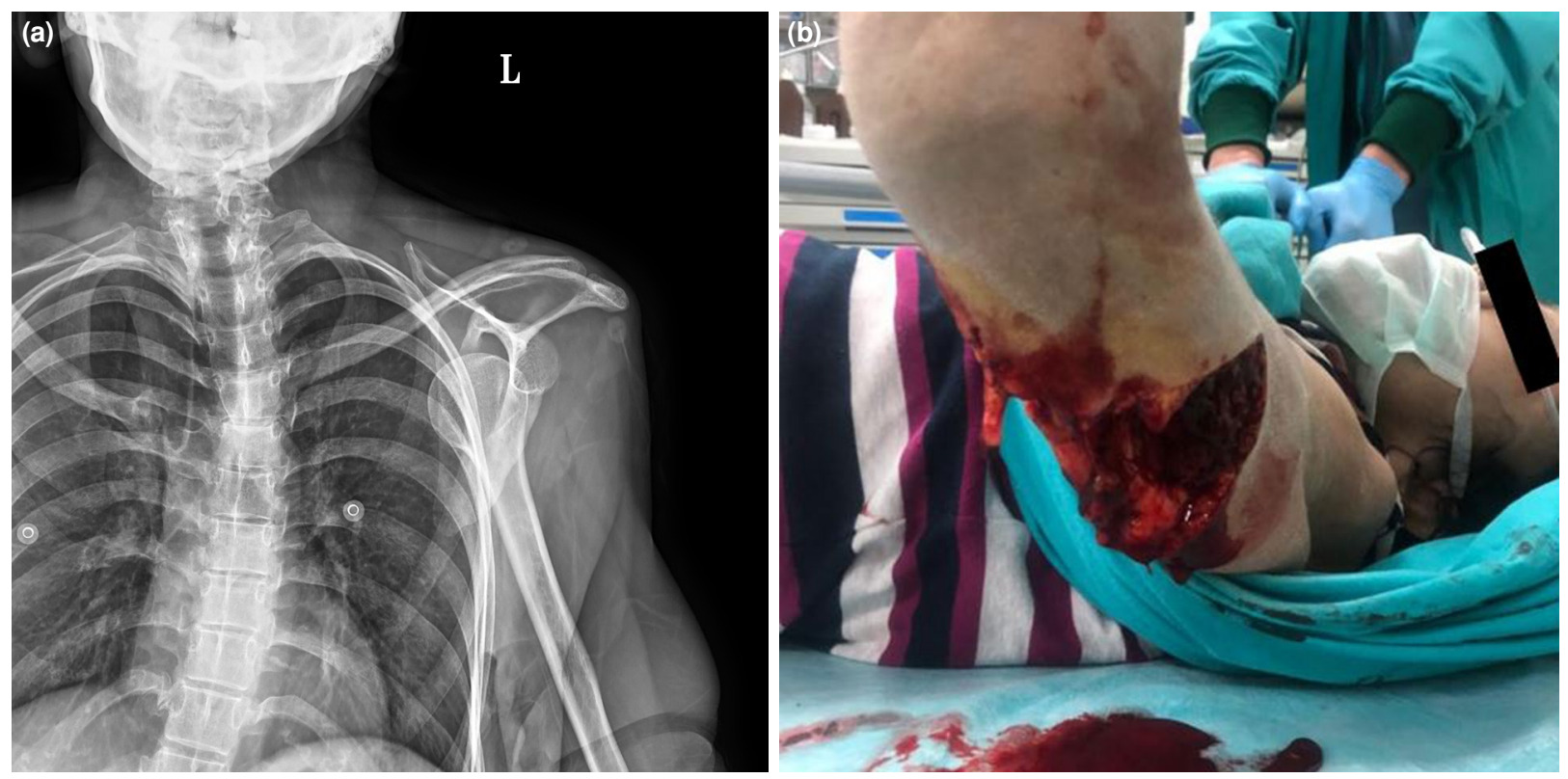

Figure 1. (a) Initial radiograph of left shoulder and, (b) clinical appearance of posterior arm. 


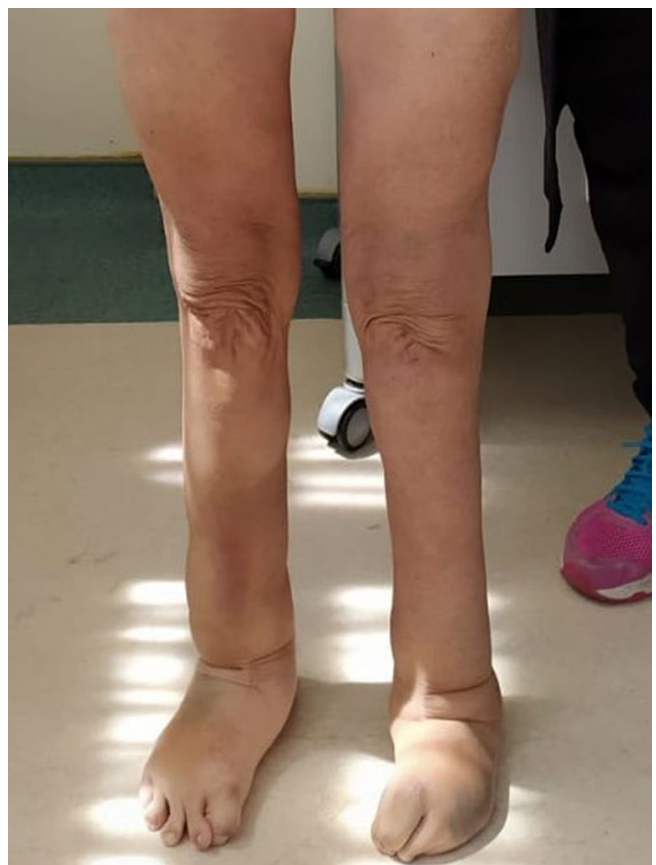

Figure 2. Characteristic lower extremity features of Larsen syndrome in our patient.

the diagnosis. Her family history revealed no features or diagnosis of LS or any other heritable syndromes. She had no history of evident congenital dislocations and she did not undergo any type of surgery. Further physical examination revealed mild-to-moderate bilateral elbow and knee recurvatum, with no signs of varus or valgus instability. Furthermore, generalized skin elasticity at the torso and the limbs was observed. Plain radiographs of all joints functioning in the appendicular skeleton were also obtained and they were found to be dysplastic, as well. She was discharged from the hospital on the same day.

The wound healed nicely after two weeks, active ROM exercises were initiated at the postoperative third week and the patient returned to the predislocation functional level at six weeks. At sixmonth and one-year follow-up, fully active and passive shoulder ROMs were observed without any signs of complications. Unfortunately, the patient was deceased due to novel coronavirus-2019 disease (COVID-19) infection, approximately 14 months after this injury and no further follow-up was possible.

As the patient was deceased, her sister was informed that data from the case would be submitted for publication and she gave her consent.

\section{DISCUSSION}

Larsen syndrome was first described in the literature in $1950 .{ }^{[4]}$ The main clinical features include multiple congenital joint dislocations at the knee, hip, and elbow joints, spinal anomalies such as scoliosis and cervical kyphosis, carpal and tarsal ossifications, supernumerary carpal bones, clubfoot, short stature; characteristic craniofacial abnormalities such as hypertelorism, prominence at the forehead, and a depressed nasal bridge. ${ }^{[3,6]}$ It is classified as a filamin group-related disease with FLNB mutations and has a dominant inheritance pattern. There are also reported sporadic forms in the literature, as in our case. ${ }^{[5,7]}$

Laville et al. ${ }^{[8]}$ reported the analysis of the 38 LS patients in their case series. They found recurrent shoulder dislocations in three patients, but further information regarding demographics, detailed physical and radiological assessments, and treatment strategies were not presented. In this study, none of the patients had a functional impairment due to the hyperlaxity of the joint "offset any problems resulting from dislocations". ${ }^{[8]}$

Bicknell et al ${ }^{[6]}$ reported clinical and radiographic features of 20 LS patients in their case series. All patients had a dislocation or subluxation in large joints (65\% elbow, $80 \%$ hip, and $80 \%$ knee). The only patient with shoulder pathology was the most mildly affected patient who had subluxable shoulders as the only large-joint symptom. ${ }^{[6]}$

Mei et al. ${ }^{[7]}$ reported a case of presumed LS in a child with a 12-year follow-up. The radiological survey of the patient revealed bilateral symmetrical shoulder subluxation at the age of 22 months. At the patient's last follow-up examination at the age of 14 , radiological findings were still present; however, the patient had neither fixed contracture nor obvious instability and was able to perform daily routines without any help; therefore, no intervention was required.

Cartault et al. ${ }^{[9]}$ reported 22 LS patients in their case series. Their series had five patients with unilateral and three with bilateral shoulder dislocations. Since their focus was on genetic studies, no further clinical information was presented.

Although LS is not considered in the hypermobility spectrum disorders, similar to Marfan and Ehlers-Danlos syndrome (EDS), it shares the typical features such as joint hypermobility and skin hyperextensibility. ${ }^{[10]}$ To the best of our knowledge, there are no published data about skin abnormalities in LS in terms of tenderness or fragility in the literature, as well as reports showing skin lacerations after shoulder joint reduction attempts in hypermobility spectrum disorders. 

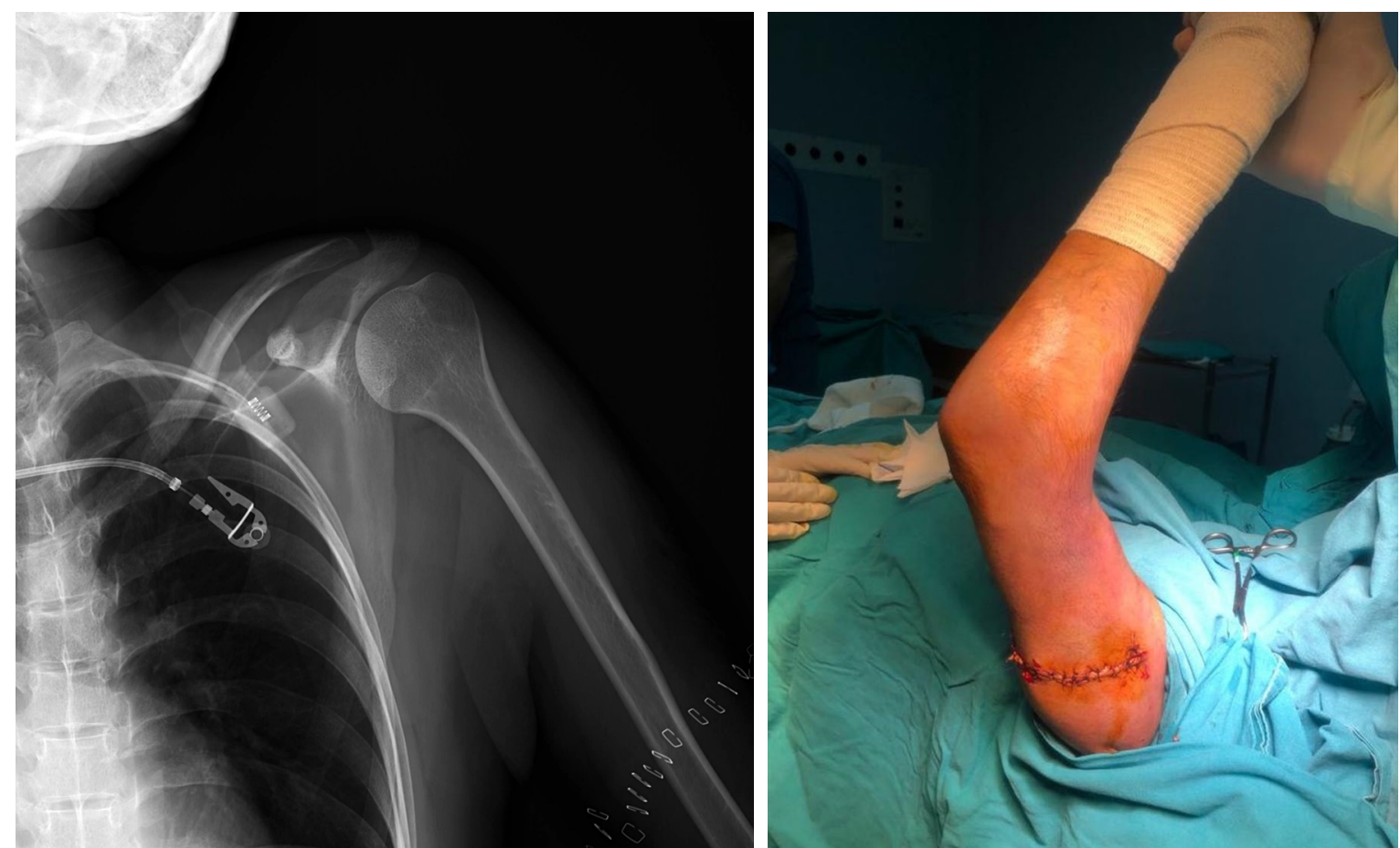

Figure 3. Radiograph and clinical appearance of left shoulder after reduction and repair of the soft tissues under sedative anesthesia.

We believe that sedation to overcome muscle strength before subsequent manipulation after the first failed reduction attempt could have prevented this deep laceration.

In conclusion, given the rarity of LS and the lack of detailed knowledge about shoulder problems among these patients, this report would be helpful to increase the awareness of orthopedic surgeons and emergency medicine physicians on this syndrome and the possibility of severe and unusual complications after TSDs. Acute TSDs in LS patients should be reduced with gentle manipulations and maneuvers, preferably under sedation or general anesthesia to prevent unusual soft tissue complications as described in this report.

\section{Declaration of conflicting interests}

The authors declared no conflicts of interest with respect to the authorship and/or publication of this article.

\section{Funding}

The authors received no financial support for the research and/or authorship of this article.

\section{REFERENCES}

1. Zacchilli MA, Owens BD. Epidemiology of shoulder dislocations presenting to emergency departments in the United States. J Bone Joint Surg [Am] 2010;92:542-9.
2. Atef A, El-Tantawy A, Gad H, Hefeda M. Prevalence of associated injuries after anterior shoulder dislocation: A prospective study. Int Orthop 2016;40:519-24.

3. Klein C, Bulaid Y, Deroussen F, Plancq MC, Printemps C, Gouron R. Congenital dislocation of the knee in a threeyear-old-child with Larsen syndrome: Treatment with a hexapod-type external fixator. Knee 2018;25:966-71.

4. Larsen LJ, Schottstaedt ER, Bost FC. Multiple congenital dislocations associated with characteristic facial abnormality. J Pediatr 1950;37:574-81.

5. Dobbs MB, Boehm S, Grange DK, Gurnett CA. Case report: Congenital knee dislocation in a patient with larsen syndrome and a novel filamin B mutation. Clin Orthop Relat Res 2008;466:1503-9.

6. Bicknell LS, Farrington-Rock C, Shafeghati Y, Rump P, Alanay Y, Alembik Y, et al. A molecular and clinical study of Larsen syndrome caused by mutations in FLNB. J Med Genet 2007;44:89-98.

7. Mei H, He R, Liu K, Wu J, Tang J, Yan A. Presumed Larsen syndrome in a child: A case with a 12-year follow-up. J Pediatr Orthop B 2015;24:268-73.

8. Laville JM, Lakermance P, Limouzy F. Larsen's syndrome: Review of the literature and analysis of thirty-eight cases. J Pediatr Orthop 1994;14:63-73.

9. Cartault F, Munier P, Jacquemont ML, Vellayoudom J, Doray B, Payet C, et al. Expanding the clinical spectrum of B4GALT7 deficiency: Homozygous p.R270C mutation with founder effect causes Larsen of Reunion Island syndrome. Eur J Hum Genet 2015;23:49-53.

10. Castori M, Hakim A. Contemporary approach to joint hypermobility and related disorders. Curr Opin Pediatr 2017;29:640-9. 